\title{
ELECTRODYNAMIC TETHERS FOR SPACECRAFT PROPULSION
}

\author{
Les Johnson \\ Mail Code PS02, NASA Marshall Space Flight Center \\ Huntsville, AL 35812 \\ 205-544-0614 (phone); 205-544-6669 (fax) \\ Robert D. Estes* and Enrico Lorenzini* \\ Smithsonian Astrophysical Observatory \\ 60 Garden Street, Cambridge, MA 02138 \\ 617-495-7261 (phone); 617-496-7670 (fax) \\ Manuel Martinez-Sanchez* \\ Massachusetts Institute of Technology \\ Cambridge, MA 02138 \\ 617-253-5613 (phone); 617-258-5143 (fax) \\ Juan Sanmartin \\ Polytechnic University of Madrid \\ Madrid, Spain \\ Irwin Vas* \\ Bosing Space and Defense Company \\ 499 Boeing Blvd., Huntsville, AL 35824 \\ 205-461-2921 (phone); 205-461-255I (fax)
}

\begin{abstract}
Abstrnet
Relatively short electrodynamic tethers can use solar power to "push" against a planetary magnetic field to achieve propulsion without expenditure of propellant. The groundwork has been laid for this type of propulsion. Recent important milestones include retrieval of a tether in space (TSS-1, 1992), successful deployment of a 20 $\mathrm{km}$-long tether in space (SEDS-1, 1993), and operation of an electrodynamic tether with tether current driven in both directions ( $P M G, 1993$ ). The planned Propulsive Small Expendable Deployer System (ProSEDS) experiment will use the flight-proven Small Expendable Deployer System (SEDS) to deploy a $5-\mathrm{km}$ bare copper tether from a Delta II upper stage to achieve $-0,4 \mathrm{~N}$ drag
\end{abstract}

thrust, thus deorbiting the stage. The experiment will use a predominantly "bare" tether for current collection in lieu of the endmass collector and insulated tether approach used on previous missions. Theory and ground-based plasma chamber testing indicate that the bare tether is a highly efficient current collector. The flight experiment is a precursor to utilization of the technology on the $\mathrm{In}$ ternational Space Station (ISS) for reboost and the electrodynamic tether upper stage demonstration mission which will be capable of orbit raising, lowering, and inclination changes-all using electrodynamic thrust. In addition, the use of this type of propulsion may be attractive for future missions to Jupiter.

\section{Electrodvnamic Tether Propulsion Principles}

An electrodynamic tether can work as a thruster because a magnetic field exerts a force on a current-carrying wire. This force is perpendicular to the wire and to the field vector. If the current flows downward through a 
tether connected to a spacecraft, the force exerted by the geomagnetic field on the system has a component that accelerates the satellite along the direction in which it is already moving.

An orbiting system, by virtue of its motion through the Earth's magnetic field, experiences an electric field $(\mathbf{v} \times \mathbf{B})$ perpendicular to its direction of motion and to the geomagnetic field vector. For an eastward-moving system, such as most Earth-orbiting spacecraft, the field is such that the electrical potential decreases with increasing altitude (at a rate of $\sim 100 \mathrm{~V} / \mathrm{km}$ for a $400-\mathrm{km}$ circular orbit). In order to drive a current down the tether, it is necessary to overcome this induced electromotive force (emf).

Thus, this propulsion system requires a power supply and may be considered a type of electrical thruster. Calculations indicate an average thrust of $0.5 \mathrm{~N}$ from $5 \mathrm{~kW}$ and $0.8 \mathrm{~N}$ from $10 \mathrm{~kW}$, assuming a tether $10 \mathrm{~km}$ long, with a mass $<200 \mathrm{~kg}$. Electrical power from solar panels could be utilized for this thruster power with night operation on battery power as an option.

A hollow cathode plasma contactor would be used on the spacecraft to eject electrons; thus, the tether must be deployed vertically downward for a boost application. Thanks to the power supply, which is placed in series between the plasma contactor and the upper end of the tether, the upper end is at a higher electrical potential than the plasma for some distance below it. This distance may be greater than the tether length if the applied voltage exceeds the motional emf. The ionospheric electrons below the spacecraft would "like" to get to the higher potential at the upper end of the tether. If the electrons can make contact with the tether, they will travel up it, giving a current flow in the correct direction for boost.

The way in which the charge exchange between tether and plasma takes place depends upon the specifics of the system, and this aspect (specifically the electron collection, which is the difficult part) is the focus in designing a system capable of producing sufficient thrust with a reasonably short tether.

The magnitude of the thrust force is dependent upon the motional emf (between the two ends of the tether), the average current in the tether, and the orbital speed, Thus, the product of the tether length and average tether current determines the thrust for given orbital/magnetic conditions. Generally speaking, a shorter tether will have a smaller impact on the spacecraft environment, so a combination of high current with short tether length is the goal.
Boost (thrust forces of order $1 \mathrm{~N}$ ) with a tether no longer than $10 \mathrm{~km}$ requires tether currents of order $10 \mathrm{~A}$. The critical issue is how to draw ionospheric electrons at that rate. The standard tether carries insulation along its entire length, exchanging current with the ionosphere only at the ends: TSS-1R carried a passive metallic sphere as anode; PMG carried an active (plasma-ejecting) contactor.

Current collected to a passive, biased sphere in a magnetized plasma calculated by the standard ParkerMurphy (PM) model (taking into account magnetic effects, which are dominant) grows as the square root of the bias voltage, an important fact for fixed-area collectors.

A preliminary analysis of the measured TSS-1R currents indicates that they were typically greater than the PM model predictions (using values of the electron density and temperature estimated from ionospheric models and a satellite voltage calculated with some uncertainty). The TSS-1R data do not, however, appear to point to a dependence of current on voltage greatly different from that of PM for higher voltages. Even though, for example, a TSS-1R current of $0.5 \mathrm{~A}$ at $350 \mathrm{~V}$ bias may surpass PM model estimates, it could still imply a voltage of roughly $35 \mathrm{kV}$ to reach $5 \mathrm{~A}$ for the same plasma parameters (which would require over $175 \mathrm{~kW}$ for a thrust of $0.7 \mathrm{~N}$ with a 10 -km-long tether!).

Active anodes (plasma contactors) have been developed in an attempt to solve both space-charge shielding and magnetic guiding effects by creating a self-regulating plasma cloud to provide quasi-neutrality and by emitting ions to counterstream attracted electrons and produce fluctuations that scatter those electrons off magnetic field lines. The only tether experiment to use an active anode so far was the $P M G$, which reached $0.3 \mathrm{~A}$ in flight under a $130-\mathrm{V}$ bias and the best ionospheric conditions. Unfortunately, there is no way to scale the results to high currents. The discouraging fact was that collected current decreased sharply with the ambient electron density at night.

Fortunately, there is another tether design optionthe bare tether. 1

\section{New Technology Tether Enhances Current Collection}

A bare-tether design represents a breakthrough that makes short-tether electrodynamic reboost with moderate power requirements practical. The tether itself, left uninsulated over the lower portion, will function as its own very efficient anode. The tether is biased positively with 
respect to the plasma along some or all of its length. The positively biased, uninsulated part of the tether then collects electrons from the plasma.

The following features argue in favor of the bare-tether concept:

1. The small cross-sectional dimension of the tether makes it a much more effective collector of electrons (per unit area) from the space plasma than is a large sphere (such as the TSS-1R satellite) at equal bias (Fig. 1). This is because the small cross-dimension of the tether allows its current collection to take place in the orbitalmotion-limited regime (OML), which gives the highest possible current density.

2. The large current-collection area is distributed along the tether itself, eliminating the need for a large, massive and/or high-drag sphere or a resource-using plasma contactor at the upper end of the tether. This substantially reduces the center of gravity shift in both cases and reduces the cost and complexity in the case of the active contactor.

3. The system is self-adjusting to changes in electron density. This is accomplished by a natural expansion of the portion of the tether that is biased positively relative to the ionosphere whenever the density drops.



Fig. 1. Current collection efficiency of the bare tether compared to a sphere of equal area.

Features (1) and (2) combine to provide an ability to collect large currents with modest input power levels below a candidate system that can produce average thrusts of $0.5-0.8 \mathrm{~N}$, for input power of $5-10 \mathrm{~kW}$.

Charged-particle collection is governed by the stronger gradients associated with the smaller dimensions and is thus a two-dimensional process, the length being irreevant to the density of current collected. For a radius, small compared to both Debye length and gyroradius, there are neither space-charge nor magnetic-guiding effects, and we are in the (OML) regime of standard Langmuir theory. In the OML regime, the current takes the largest possible value for the given geometry and bias. Better still, it turns out that in cylindrical geometry, the OML regime holds for radius-to-Debye length ratios even of order unity. Hence, a cylinder of 5-mm radius (about one Debye length, and small compared with gyroradius) works in the OML regime.

For a cylinder of $2-\mathrm{mm}$ radius and $2.5-\mathrm{km}$ length in a plasma with an electron density and temperature of $10^{12} \mathrm{~m}^{3}$ and $0.15 \mathrm{eV}$, respectively, the bias voltage required to collect $10 \mathrm{~A}$ is only $100 \mathrm{~V}$ ! But a tether is just a long, thin cylinder. Thus, if left uninsulated along part of its length, a tether can act as its own anode, capturing electrons efficiently over some positively biased segment.

For an orbiting, current-carrying tether, the bias will actually vary along the tether because of both the motional electric field and the ohmic voltage drop. The electron current to the tether will thus vary with height. Along the uninsulated part of the tether, the tether current will decrease with decreasing altitude, until the point is reached at which the tether is at zero bias with respect to the plasma (or the end of the tether is reached). Assuming there is a point of zero bias on the tether, then below that point an ion current (much smaller because of the high ratio of ion mass to clectron mass) that decreases somewhat the average tether current will be collected, due to the negative bias.

The bias required to collect a given OML current varies as the inverse square of the collecting area, making it possible to reduce the required bias substantially by modestly increasing the collecting area. Since the current collected by an electron-collecting length $L_{B}$ grows roughly as $\left(L_{B}\right)^{3 / 2}$, the tether can automatically accommodate drops in density by increasing the length of the collecting segment, shifting the zero bias point downward. Figure 2 shows the variation in thrust with electron density for a $10-\mathrm{km}$ tether with a 5 -km-long bare segment. Thrust drops only 10 percent as density drops by a factor of 10. The reason is clear: the collecting length has increased from 1 to $4 \mathrm{~km}$ (emf is $1,200 \mathrm{~V}$; input power 10 $\mathrm{kW}$ ). This ability to maintain thrust levels with low electron densities makes nighttime boost possible. 


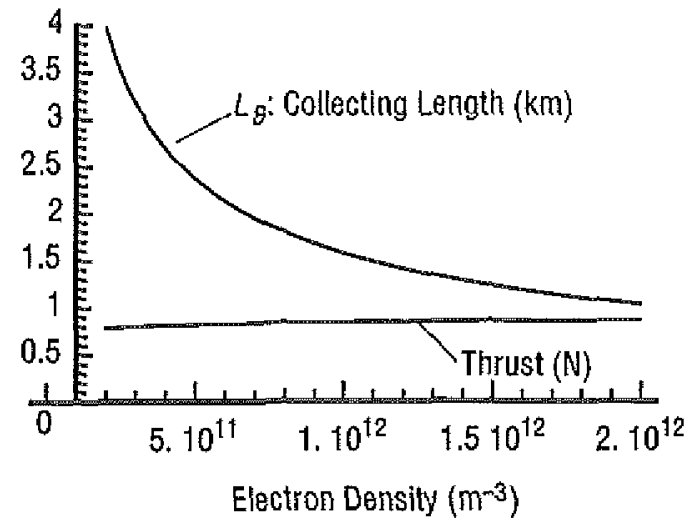

Fig. 2. Variation in thrust with electron density for a $10 \mathrm{~km}$ tether with a 5 -km-long bare segment.

Another important fact is that the OML current is identical for all cylinders with convex cross sections of equal perimeter: ${ }^{2}$ With maximum crosswise dimension (here about $10 \mathrm{~mm}$ ) fixed by OML considerations, one is allowed to choose the cross-sectional shape. This frees us to choose a tape or ribbonlike tether, for example. The tapelike geometry gives somewhat better performance than a circular cross-section tether of equal length and mass and appears to have advantages related to deployment and thermal concerns.

On the whole, the simplicity of the design, in addition to the ability to collect high currents and to accommodate density fluctuations by varying the collecting area, make the bare-tether concept particularly attractive. Bare tethers ate mostly free of the gross perfomance uncertainties that cloud the use of active, or spherelike passive, contactors. The OML theory has been substantiated for both quiescent and flowing plasmas in the laboratory, and also in rocket and satellite flights, at moderate voltages. ${ }^{3-5}$

Ground simulation of electron collection in orbital conditions is possible because there is no need to reproduce the cylinder length-to-radius ratio in the lab; also, the orbital velocity should have no effect at the low radius-to-gyroradius ratio of interest. A series of plasma chamber tests were conducted at the NASA Marshall Space Flight Center in the spring of 1997 with promising results. Figure 1 shows the current collection efficiency of the bare tether compared to a sphere of equal area. The two-dimensional geometry also makes a largescale program of particle-in-cell simulations feasible, and we anticipate using such simulations to study various tether geometries in our search for increased performance with lower mass.

\section{Technology Applications}

\section{Reboost of the ISS}

A concept design for an electrodynamic tether thruster capable of delivering $0.5-0.8 \mathrm{~N}$ of thrust to the $I S S$ at a cost of $5-10 \mathrm{~kW}$ of electrical power consists of a $10-\mathrm{km}$-long aluminum tether in the form of a thick ribbon $(0.6 \mathrm{~mm}$ by $10 \mathrm{~mm})$. Despite its length, the tether would weigh only around $200 \mathrm{~kg}$. Since the bare portion of the tether is to act as the electron collector, a downward deployment of the tether is dictated by the physics of the eastward-moving platform.

The upper part of the tether will be insulated. There are two reasons for this. First, there is the necessity for preventing electrical contact from developing across the plasma between the upper portion of the tether and the Space Station, which (when the system is operating) are separated by an electrical potential difference of around a kilovolt. Secondly, the insulation provides for greater thrust at a given input power. This comes from the fact that the largest tether-to-plasma bias occurs at the upper end, and decreases down the tether. A completely bare tether would draw the maximum current through the power supply, but the current would be strongly peaked at the upper end of the tether. Keeping the input power constant, we can substantially increase the average current in the fether, and hence the thrust, by insulating the tether over much of its upper portion, collecting current with the lower portion, and having a constant current in the upper part.

Determining the optimal fraction to insulate is part of the design effort for a "bare" tether reboost system. The preliminary design has the upper 50 percent of the tether insulated. Even greater thrust during daytime operation could be obtained with a higher fraction, but the nighttime adjustability would suffer.

The system provides flexibility in the sense that the thrust obtained depends almost linearly on the input power, as seen in Figure 3.

The bare-tether design has essentially "cured" the problem of day/night thrust fluctuations. But fluctuations in thrust due to fluctuations in the induced emf as the system encounters a varying geomagnetic field around the orbit are a fact of life for any tether-based system. Figure 4 shows variations in thrust around two typical revolutions of the ISS orbit for the $10 \mathrm{~km}$-long electrodynamic tether thruster described in this section, as it operates at a constant power of $10 \mathrm{~kW}$. Dependence on electron density is weak, as expected. Thrust curve basically 
tracks emf. Figure 5 shows a comparison of thrust generated for input powers of $5 \mathrm{~kW}$ and $10 \mathrm{~kW}$ for same tether and orbit as Figure 3.



Fig. 3. Variation of thrust with input power for nominal $10-\mathrm{km}$ system. Motional emf: $1.2 \mathrm{kV}$.

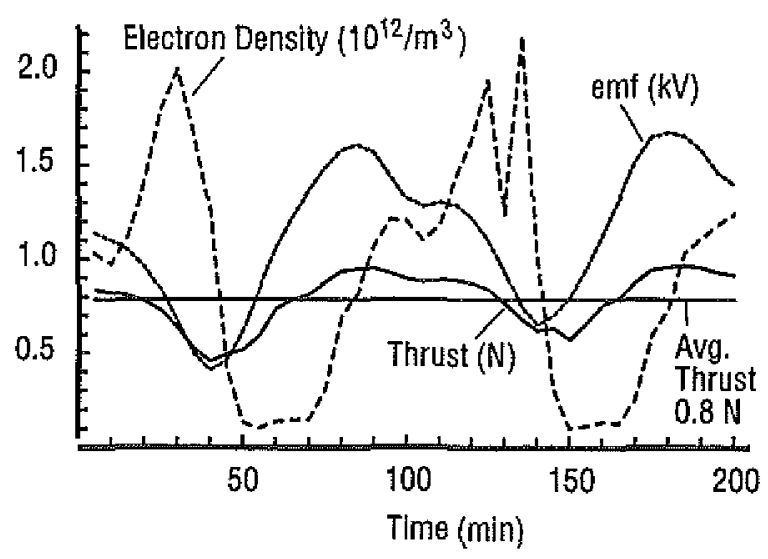

Fig. 4. Comparison of thrust operating at a constant power of $10 \mathrm{~kW}$.



Fig. 5. Comparison of thrust generated for input powers of $5 \mathrm{~kW}$ and $10 \mathrm{~kW}$.
Given the level of the current the system may draw, the system will almost certainly require its own cathodic plasma contactor at the Station end. The contactors currently under development at NASA Lewis Research Center should be well suited for this function. If thrusts $>0.5 \mathrm{~N}$ are desired, it is likely that the system will also have to rely on the $I S S$ 's plasma contactor as well, or on a second dedicated contactor, since currents over the 10-A rating of the contactors could be required.

The value in an electrodynamic tether reboost system lies in its ability to couple power generation with thrust. Heretofore, the electrical and propulsion systems have been effectively totally separate entities. Outfitting ISS with an electrodynamic reboost tether severs the most critical and constraining dependency on Earth-propellant resupply. The Station can supply its own power but not its own propellant. Without an electrodynamic tether, the specter of SkyLab and the words "reentry" and "atmospheric burnup" will forever haunt the minds of anyone who has an interest in the program. Add a tether and some additional storage capacity for supplies, and suddenly a 1-year interval between visits to the Station becomes conceivable.

Even if the current frequency of resupply flights to the Station is maintained, with an electrodynamic tether the Station Program has the option to trade kilowatts for increased payload capacity. Resupply vehicles can deliver useful cargo like payloads, replacement parts, and crew supplies rather than propellant. Within the range of 5 to $10 \mathrm{~kW}$, a crude approximation of $1,000 \mathrm{~kg}$ of user payload gained per kilowatt expended per year appears reasonable.

Yet another dimension to propellantless reboost must be considered. Station users have been allocated a minimum of 180 days of microgravity per year. Cument planning essentially halts science activity during reboost maneuvers. Low-thrust, electrodynamic tether reboost could be perfomed over long duration, as opposed to shott-duration, highthrust propulsive maneuvers. The $0.5-0.8 \mathrm{~N}$ thrust provided by a $10-\mathrm{km}$ tether more than counteracts the Station's atmospheric drag on a daily basis. Recent analysis indicates that an electrodynamic tether can compensate for the drag while it is occurning, without disrupting the microgravity environment. Fluctuations in the induced voltages from the Earth's magnetic field and in electron densities will create "turbulence" through which the electrodynamic tether-driven Station must fly; can load-leveling control systems compensate for these pockets and maintain microgravity levels? In this case, a new realm of possibilities opens up for long-duration microgravity experiments. The allure of this self-propelled space facility is certainly remarkable, and offers potential advantages. 


\section{Reusable Upper Stage Propulsion}

An electrodynamic upper stage could be used as an orbital tug to move payloads in low-Earth orbit (LEO) after launch from a Reusable Launch Vehicle or other launch vehicle. The tug would rendezvous with the payload and launch vehicle, dock/grapple the payload, and maneuver it to a new orbital altitude or inclination within LEO without the use of boost propellant. The tug could then lower its orbit to rendezvous with the next payload and repeat the process. Such a system could conceivably perform several orbital maneuvering assignments without resupply, making it a low recuring cost space asset. The performance of a $10 \mathrm{~kW}, 10-\mathrm{km}$ tether system is i1lustrated in Figures 6 and 7. The performance of an electrodynamic tether thruster varies with altitude in the magnetosphere (where $i$ is the orbital inclination) (see Fig. 6). Electrodynamic tethers can be used to change orbital inclination without propellant consumption. To determine the available inclination change for a spacecraft/payload mass, divide the "specific inclination tate" indicated by the total system mass at a given altitude (see Fig. 7).

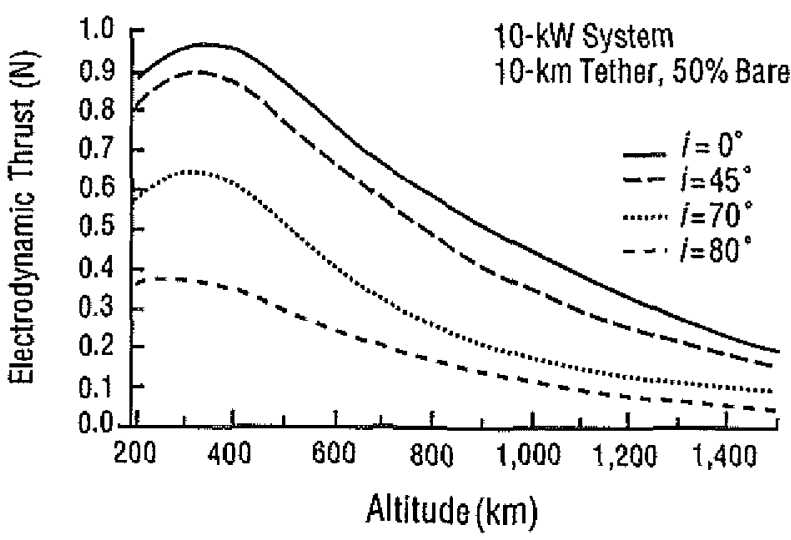

Fig. 6. Performance of an electrodynamic tether thruster:

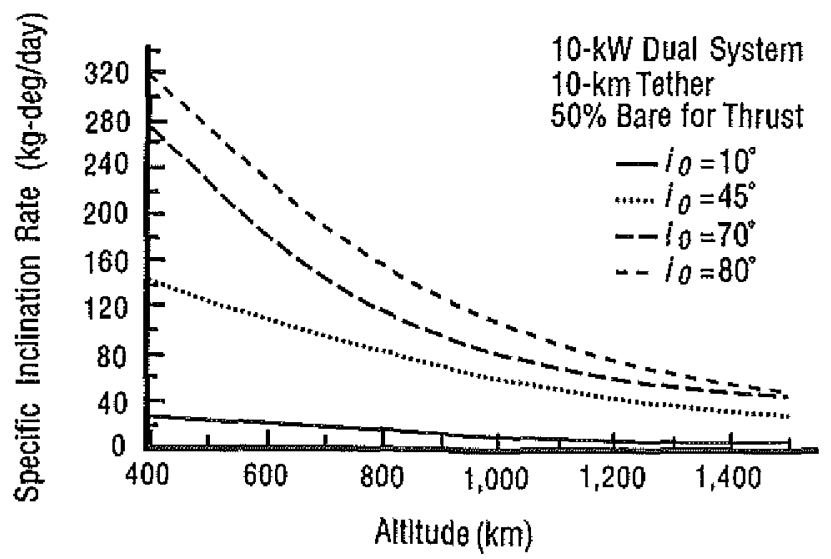

Fig. 7. Electrodynamic tethers change orbital inclination without propellant consumption.

\section{Jovian Exploration}

Following the successful Galileo mission, there is considerable interest in a follow-on mission to Jupiter and its moon, Europa. Due to low solar luminosity Sun, radioactive thermoelectric generators (RTG's) were used for electrical power by Galileo and in all past deep space missions. The finite risk of releasing plutonium into the terrestrial environment may rule out RTG's on future missions. The possibility of using solar panels for electrical power generation has improved in recent years with improvements in this technology. The high levels of radiation in the Jovian system, however, are expected to rapidly degrade the effectiveness of solar arrays as a result of extended exposure. Extended operations in the Jovian system, or around any planet, also typically require use of an expendable propellant for orbital maneuvering. This may lead to high "wet" spacecraft mass at launch and/or limited lifetime on orbit. It is for these reasons and because of the strong magnetic field and rapid planetary rotation that electromagnetic tethers are being considered for use in the Jovian magnetosphere. Preliminary analysis indicates that a megawatt of power can be theoretically generated by a $10-\mathrm{km}$ tether in near Jovian space (see Fig.8). Specifically, such a tether operating near the planet would experience induced voltages greater than $50,000 \mathrm{~V}$, currents in excess of $20 \mathrm{~A}$, generate approximately $1 \mathrm{MW}$ of power and experience more than $50 \mathrm{~N}$ of thrust! ${ }^{6}$ Needless to say, this would pose significant engineering challenges for mission planners.

Power (W)

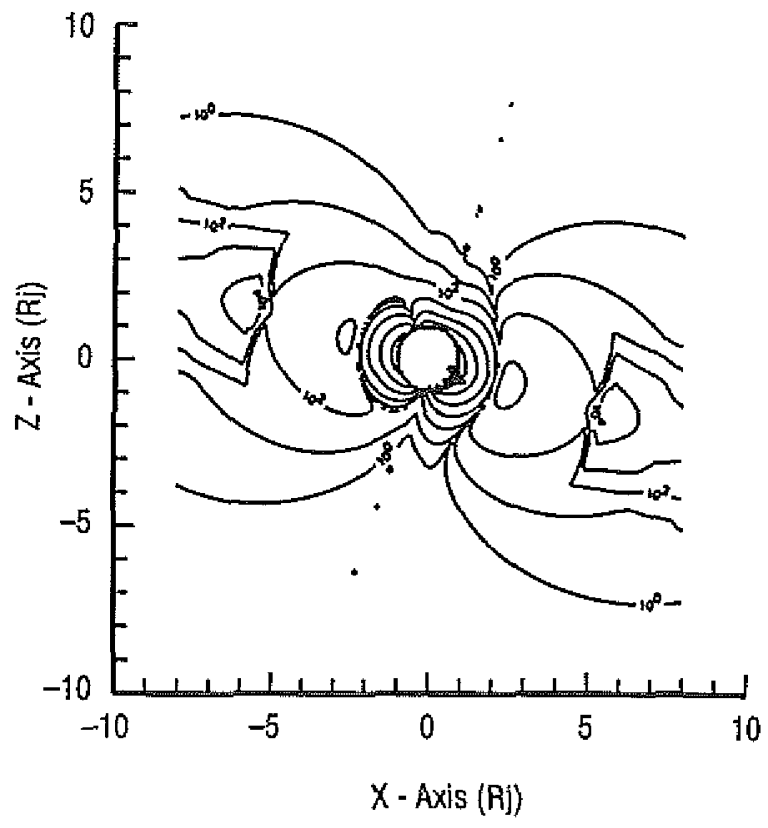

Fig. 8. Power generated in a 10-km tether at Jupiter. Contours are drawn for even decades of power from $1 \mathrm{~W}$ to $10 \mathrm{MW}$. 


\section{ProSEDS Flight Experiment}

A flight experiment to validate the performance of the bare electrodynamic tether in space and demonstrate its capability to perform thrust is planned by NASA for the year $2000 .^{7}$ The ProSEDS experiment will be placed into a $500-$ $\mathrm{km}$ circular orbit as a secondary payload from a Delta II launch vehicle. The flight-proven SEDS will be used to deploy a $5-\mathrm{km}$ predominantly bare copper wire attached to 20 $\mathrm{km}$ of insulating Spectra tether and $25-\mathrm{kg}$ endmass. The deployer and endmass mounted on the Delta II upper stage are shown in Figure 9.

Delta ll Second Stage

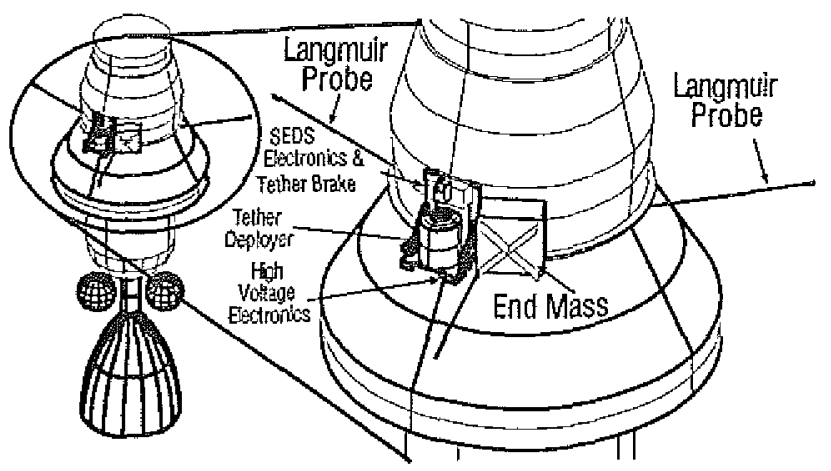

Fig. 9. ProSEDS experiment hardware on the Delta II upper stage. 8
Once on orbit, the SEDS will reel out the tether and endmass system to a total length of $25 \mathrm{~km}$. Upward deployment will set the system to operate in the generator mode, thus producing drag thrust and producing electrical power. The drag thrust provided by the tether will deorbit the Delta II upper stage in approximately 3 weeks, versus its nominal $1.5-\mathrm{yr}$ lifetime in a $500-\mathrm{km}$ circular orbit. Approximately $100 \mathrm{~W}$ electrical power will be extracted from the tether to recharge mission batteries and to allow extended measurements of the system's performance until it reenters.

\section{Conclusions}

Tether technology has advanced significantly since its inception over 30 years ago. The recent successes of the SEDS system shows that tethers are ready to move from experiment and demonstration to application. One of the most promising applications for tethers is space propulsion. The use of electrodynamic tether propulsion for reusable upper stages, planetary missions, space station, and launch vehicle deorbit applications will soon be demonstrated with the ProSEDS experiment. 


\section{References}

1. Sanmartin, J.R., Martinez-Sanchez, M. and Ahedo, E,, "Bare wire anodes for electrodynamic tethers," $J$. Propulsion and Power, Vol. 9, 1993, p. 353-360.

2. Laframboise, J.G., and Parker, L.W., "Probe design for orbit-limited current collection," "Phys. Fluids, Vol. 16,1973 , p. $629-636$.

3. Chung, P.M., Talbot, L., and Touryan, K.J., "Electric Probes in Stationary and Flowing Plasmas: Theory and Application," Springer-Verlag, NY, 1975.

4. Mercure, H.P.E., "Ion temperature measurement in a flowing collisionless plasma using an end effect of cylindrical Langmuir probes"University of Toronto Inst. for Aerospace Studies, Report No. 202, July 1976.

5. Szuszczewicz, E.P., and Takacs P.Z., "Magnetosheath Effects on Cylindrical Langmuir Probes," Phys. Fluids, Vol. 22, 1979, p. 2424-2429.
6. Gallagher, D.L., Bagenal, F., Moore, J., and Johnson, L., "An Overview of Electrodynamic Tether Performance in the Jovian System," Proceedings of the 1997 NASA Tether Technology Interchange Meeting, Huntsville, AL, September 1997.

7. Johnson, L., and Ballance, J., "The Propulsive Small Expendable Deployer System (ProSEDS) Experiment," Proceedings of the 1997 NASA Tether Technology Interchange Meeting, Huntsville, AL, September 1997.

8. Hajos, G., Unpublished drawing provided for the NASA Ptopulsive Small Expendable Deployer System (ProSEDS) project, NASA Marshall Space Flight Center, Huntsville, AL, 1997. 